OPEN ACCESS

Edited by: Ingrid Melle,

University of Oslo, Norway

Reviewed by:

Michael Compton,

Columbia University, United States

Johannes Langeveld,

Stavanger University Hospital, Norway

Inge Joa,

Stavanger University Hospital, Norway

*Correspondence:

Sara Farhang

s.farhang@umcg.n

tThese authors have contributed equally to this work

Specialty section:

This article was submitted to

Schizophrenia

a section of the journal

Frontiers in Psychiatry

Received: 10 November 2020

Accepted: 21 January 2021

Published: 10 February 2021

Citation:

Farhang S, Ghaemmaghami M, Shafiee-Kandjani AR, Noorazar SG,

Veling W, Malek A, Somi MH, Bruggeman R and Alizadeh BZ (2021)

An Observational Cohort of First

Episode Psychosis in Iran: The Azeri Recent Onset Acute Phase Psychosis Survey (ARAS Cohort) Study Protocol.

Front. Psychiatry 12:627960.

doi: 10.3389/fpsyt.2021.627960

\section{An Observational Cohort of First Episode Psychosis in Iran: The Azeri Recent Onset Acute Phase Psychosis Survey (ARAS Cohort) Study Protocol}

\author{
Sara Farhang ${ }^{1,2 \star}$, Mehrdad Ghaemmaghami $^{2}$, Ali Reza Shafiee-Kandjani ${ }^{2,3}$, \\ Seyed Gholamreza Noorazar ${ }^{2}$, Wim Veling ${ }^{4}$, Ayyoub Malek ${ }^{2}$, Mohammad Hossein Somi ${ }^{5}$, \\ Richard Bruggeman ${ }^{1 \dagger}$ and Behrooz Z. Alizadeh ${ }^{1,6+}$ \\ ${ }^{1}$ University Medical Center Groningen, University Center for Psychiatry, Rob Giel Research Center, University of Groningen, \\ Groningen, Netherlands, ${ }^{2}$ Research Center of Psychiatry and Behavioral Sciences, Tabriz University of Medical Sciences, \\ Tabriz, Iran, ${ }^{3}$ Faculty of Medicine, Tabriz University of Medical Sciences, Tabriz, Iran, ${ }^{4}$ Department of Psychiatry, University \\ Medical Center Groningen, University of Groningen, Groningen, Netherlands, ${ }^{5}$ Liver and Gastrointestinal Diseases Research \\ Center, Tabriz University of Medical Sciences, Tabriz, Iran, ${ }^{6}$ Department of Epidemiology, University Medical Center \\ Groningen, University of Groningen, Groningen, Netherlands
}

Background: Most of our knowledge about the etiology, course, treatment, and outcome of schizophrenia spectrum and other psychotic disorders stems from Western countries. Data from populations living in other geographical areas and low- and middle-income countries, with different genomes (ethnicity) and exposomes (e.g., culture and social support, drugs of abuse, religion), will add to our knowledge of this complex disorder.

Methods: The Azeri Acute phase/Recent onset psychosis Survey (ARAS) has been initiated to study the course of the disorder in patients with recent-onset psychosis using validated diagnostic tools and a comprehensive outcome monitoring system, aiming to reveal indicators for understanding the risk and resilience factors and for choosing the best-personalized treatment strategy. All participants will be evaluated for clinical signs and symptoms as well as risk and resilience factors and will be followed up for 1, 3, and 5 years for outcomes in several domains. A hierarchical cluster method will be applied to identify the number of clusters for each outcome. Defined models will be applied to assess the predictive value of cognition on symptomatic and functional outcomes at follow-up.

Discussion: The ARAS cohort will yield significant academic- (research and education) and care-related achievements. ARAS data and experience will have value both in being a useful model for other parts of this region and in an expansion of the currently available knowledge.

Keywords: schizophrenia, first episode psychosis (FEP), social context, cognition, gene-environement interactions 


\section{BACKGROUND}

Schizophrenia, as the prototype of the schizophrenia spectrum (SSD) and other psychotic disorders (1), remains one of the most costly disorders in terms of human suffering and societal expenditure $(2,3)$. It contributed $1.7 \%$ of total years of life lived with disability (YLDs) to the global burden of disease in 2016. The incidence of schizophrenia varies across and within countries, as a wide distribution range is noticeable in available studies $(4,5)$. A systematic review of 161 studies reported a median incidence rate of 15.2 per 100,000 , with significant effects of migrant status, gender, and urbanicity $(6,7)$. There have also been differences noted in point and lifetime prevalence estimates related to economic status and latitude $(8,9)$. The course of illness may also differ across contexts. Older studies suggested that the course of psychotic disorders may be more favorable in lowand middle-income countries (LMIC) (WHO 10 country study), while other studies point at a large treatment gap as a cause of poor outcome in LMIC (10). Socio-cultural differences such as social support, religious and cultural beliefs, and differences in mental health care organization might explain a substantial portion of these epidemiological variations (11).

SSDs and other psychotic disorders are characterized by positive symptoms including hallucinations and delusions, negative symptoms such as apathy and social withdrawal, and cognitive impairment. Somatic comorbidity shows a high prevalence, of which the most common types include metabolic syndrome, diabetes mellitus, and cardiovascular diseases (12), adding to a remarkable increase in the rate of all-cause mortality in recent decades (9).

SSDs are considered complex genetic disorders. The heterogeneous clinical presentation most likely results from a variety of gene-environment interactions (13). A wide body of evidence suggests that highly dynamic multi-dimensional interactions between small effects of the genome and exposome underlie the etiopathogenesis of SSDs $(13,14)$. The exposome encompasses environmental factors, such as social, physical, chemical, biological, and behavioral patterns, over a lifetime, which can all vary widely between populations, in a similar manner to the genome (15).

Antipsychotic medication, cognitive-behavioral therapy, and rehabilitation are the cornerstones of treatment. Antipsychotic medications, the underlying illness, and their interaction increase the risk of cardiometabolic abnormalities in patients with SSD (16-18). Here, lifestyle and diet affect this process, and, again, these might differ substantially between populations.

Of note, incomplete response to pharmacotherapy is seen in $30-40 \%$ of patients (19). This proportion has remained almost unchanged over time, despite several decades of pharmacological improvements, and the discovery of new medications (20). Meanwhile, studies continue to examine indicators for a better response to a specific medication or liability for side effects (21). However, the predictive value of this personalized approach still needs to be increased before it can be applied to clinical practice.

Despite the differences in genomes, exposomes, and lifestyles across populations, the main volume of the current knowledge about risk and resilience factors for SSD has been collected in high-income Western countries. Recently, some studies from Asia have enriched the current knowledge concerning the role of diverse ethnicity and different living situations on SSD $(22,23)$. Beyond ethnicity, factors such as childhood adversity, trauma, use of illicit drugs, neurocognitive abilities, social and familial support, and genetic factors all constitute wellestablished modulators of SSD. So far, there are very limited data on SSD from the Middle East and Central Asia, including Iran, and specifically from populations with Turkish heritage. Thus, little is known about the attribution of environmental factors and genetics to the development of SSD in Middle Eastern and Central Asian populations. Given differences in genetics, culture, lifestyle, and population beliefs in these middle- and low-income countries, such as Iran, it is expected that risk and resilience factors, the magnitude of their interaction, as well as the course of SSD are different from those of Western, high-income countries. Investigating these differences between populations and their roles in the development and course of SSD provides a unique opportunity to understand the full spectrum of factors involved in the etiology and pathogenesis of SSD and other psychotic disorders.

Diagnostic procedures, treatment, and rehabilitation in Iran are based on well-known international diagnostic classification systems and up-to-date western guidelines (24). Nevertheless, serious gaps in in-depth research with a focus on the causes, management, and consequences of SSD exist in this population $(10,25,26)$. Looking at Iran as an example of a naturally rich, but low-income country, we previously observed patients with SSD and their families facing a variety of social, familial, and economic challenges $(27,28)$.

Accurate knowledge on the burden of SSD on the personal, societal, and health care systems is lacking, as comprehensive national registry data are not yet available. For the same reason, little is known about the outcome of Iranian patients with SSD. It can be argued that the "real-world" implementation of evidencebased practices will be hampered by social and economic factors resulting in sub-optimal care of Iranian patients. Therefore, it is essential to establish well-designed sufficiently large longitudinal studies to investigate the risk and resilience factors involved in SSD in Middle East regions. We, therefore, initiated the Azeri Recent onset/Acute phase psychosis Survey (ARAS), which investigates the course of recent-onset psychosis by using validated diagnostic tools, setting up a comprehensive outcome monitoring system, aiming to reveal indicators for understanding the risk and resilience factors associated with SSD, and for choosing the best-personalized treatment strategy. Next, to improve mental health care, the ARAS-project will define and validate the first population-specific guideline for the management of SSD in Iran. Participants will be followed up at years 1,3 , and 5 after recruitment by face to face interview using structured validated questionnaires. The specific objectives of this study are to (I) determine the disorder course regarding clinical and social aspects and predefined its subtypes, (II) identify genetic and environmental risk and resilience factors, (III) investigate whether SSD subtypes of clinical and social aspects can predict patient prognosis, and (IV) integrate findings 
from objectives I to III to build patient-centered management of psychosis.

\section{METHODS}

\section{Study Population}

The catchment area for this observational cohort study is East Azerbaijan, a province in the North of Iran with more than 3, 7 mln inhabitants, with the majority having an Azeri ethnic background. Iranian Azeris are the largest minority in the country with a Caucasian ethnic background This community is the world's largest Azeri population who are a Turkic ethnic group. The dominant religion in this region is Islam, followed by Christianity, Zartosht, and Jewish.

\section{Eligibility Criteria}

The study target population includes all inhabitants of East Azerbaijan province who are referred with (signs of) a first psychotic episode or evaluated for a recurrent psychotic episode that was previously undiagnosed ( $<2$ years). The cohort will include patients with a diagnosis of schizophrenia, schizophreniform disorder, delusional disorder, brief psychotic disorder, schizotypal personality disorder, schizoaffective disorder, or substance-induced psychotic disorder based on the Diagnostic and Statistical Manual of Mental Disorders (DSM)-5 (1). Patients receiving any of these diagnoses within the 2 years prior to the inclusion date, will be invited to participate in the study. A patient will be included only after giving written informed consent. If a patient is mentally incapable of giving informed consent, as considered by the study clinicians, this patient will be either excluded or the patient's first-degree relatives or attorney will be requested to sign the informed consent. There will be no other exclusion criteria.

\section{Study Set-Up}

The ARAS project was approved by the ethical committee of the National Institute for Medical Research Development (NIMAD) in Iran in 2017 (record number: IR.NIMAD.REC.1396.101). All study procedures will be carried out according to the Declaration of Helsinki. Written informed consent will be obtained from all participants and their caregivers or legal guardians. Patients can leave the study at any time for any reason with no influence on their quality of care. A code will be given to each patient and all data will be recorded anonymously. The participants will be informed that all study findings will be stored and handled in strict confidence according to national guidelines. The principal investigator and the main investigators have access to the materials.

There have been several meetings with psychiatrists and psychologists all over the province to describe the aim of this study. With the incentive of a free, standard, and comprehensive evaluation of their patients, they were invited to refer their patients for participation. Reports are sent back to the referring colleague in all of the sessions along with any suggestion for additional workups. Travel costs are reimbursed for patients, and waiting room refreshments were provided during assessments.
This project started in January 2018. For the first baseline assessment (referred to as T0, Figure 1), clinical interviews and assessments will take place at the treating clinics, starting at the time of admission or as soon as possible if forced admission is required. Psychological testing will be performed within 1 month after inclusion. Diagnostic interviews, questionnaires, and other instruments will be conducted in Farsi, the official language of the country.

Upcoming assessment as routine outcome monitoring will be scheduled for all patients at the 1 year follow up (T1), which will include the same assessments used in T0, including outcome measurements and assessment for direct costs. The second follow-up (T3) is scheduled at 3 years and the final followup assessment at 5 years (T5) after inclusion. Treatment strategy and visit intervals will be defined by the treating psychiatrist between inclusion and follow-up periods.

\section{Assessments}

Assessments will be performed in separate meetings depending on their preferred schedule (Figure 1). Clinical assessments will be performed by test psychologists/psychiatrists who are trained by repeated workshops.

\section{Clinical Diagnosis and Biography}

Clinical diagnoses will be made by referring psychiatrists from in- and out-patient clinics. The clinical diagnosis within the SSD will be confirmed by the Structured Clinical Interview for DSM-5 (SCID) (29), or for patients $<18$ years by the KiddieSchedule for Affective Disorders and Schizophrenia Present and Lifetime Versions (K-SADS-PL) (30). Farsi versions of these two questionnaires have been used by several research teams inside Iran and contain few cultural adaptations (31). In terms of a discrepancy between the diagnosis made by the treating clinician and researcher-administered interview, athird expert opinion from an independent psychiatrist is requested. For special cases, a panel of experts will discuss the patients' profiles to arrive at a consensus. The referring clinician will be informed about the outcome and if the patient will be included.

Dimensions of Psychosis will be rated using the symptom severity dimension tool of DSM-5 (32). The severity of symptoms will be rated using the Positive and Negative Syndrome Scale (PANSS), which is a semi-structured interview on the symptom severity including three subscales of positive and negative symptoms and general psychopathology (33). The severity of depressive symptoms will be measured using the Calgary Depression Scale in Schizophrenia (CDSS) (34). This instrument differentiates between depression and the negative and positive symptoms of schizophrenia (35).

Side effects of antipsychotics and the patients' adherence to their medication will be assessed using the Subject's Response to Antipsychotics (SRA-34) (36). Evaluation for hyperkinesia, parkinsonism, akathisia, and dystonia will be performed using the St. Hans Rating Scale (SHRS) (37).

A medical history will be obtained from the patient and the first-degree relatives of patient. The nature, severity, and consequences of symptoms for daily functioning will be recorded, and, besides their medication-history, nicotine, illicit drug, and 


\begin{tabular}{|c|c|c|c|c|}
\hline & \multicolumn{2}{|c|}{ Enrolment } & \multicolumn{2}{|c|}{ Follow up } \\
\hline & 0 & $T 1$ & $T 3$ & T5 \\
\hline Structured psychiatric interview & $\mathrm{x}$ & $x$ & $x$ & $x$ \\
\hline Physical examination & $x$ & & & \\
\hline laboratory tests & $x$ & & $x$ & $\mathrm{x}$ \\
\hline Informed consent & $x$ & & & \\
\hline Blood sampling & & $x$ & & \\
\hline \multicolumn{5}{|l|}{ Assessments: } \\
\hline Risk and resilient factors & & $x$ & & \\
\hline Clinical symptoms & & $x$ & $x$ & $x$ \\
\hline Level of functioning & & $x$ & $x$ & $x$ \\
\hline Cognitive performance & & $x$ & $x$ & $x$ \\
\hline Medication side effect & & & $x$ & $x$ \\
\hline Received care & & & $x$ & $\mathrm{x}$ \\
\hline
\end{tabular}

FIGURE 1 | The schedule of enrolment, assessments and follow-up in the ARAS study.

alcohol use, and history of illness in the family will be recorded. The biography focuses on signs of an early developmental disorder, current living situation, occupation and daytime activities, and social interactions.

\section{Medical Assessments}

A physical health status examination, including a physical examination and measurement of blood pressure, heart rate, height, weight, and waist circumference will be carried out. Laboratory tests will be conducted to test for general health conditions, including complete blood cell counts, lipid profiles, fasting blood sugar and glycohemoglobin A1c (HbA1c), liver function tests, kidney function tests, and pituitary function tests, and urine analysis for drugs including morphine, amphetamine, cannabis, tramadol, methadone and presence of abnormal routin tests. When clinically indicated, additional examination will be performed such as brain imaging, electroencephalography, or electrocardiography by the treating psychiatrists. The requirement for external consultation for any general medical condition will be decided by the psychiatrist and results will be recorded.

\section{Risk and Resilience Factors}

The evaluation process will continue with semi-structured interviews and self-report questionnaires in different domains. History of experienced adversities during early life and peer interaction in primary school will be evaluated using the Illinois Bully Scale (38), Olweus Bully/Victim Questionnaire (39), and the Retrospective bullying experience (40). The Illinois Bully Scale includes items that address how often youth is engaged in social aggression, physical, and verbal types of victimization and physical fighting with peers (34). The Olweus Bully/Victim Questionnaire includes the initiation of an act of bullying against the participant, as well as the expression of bullying behavior against others. The questions include several aspects of bully/victim problems including physical, verbal, indirect, racial, and sexual bullying annoyance. The questionnaire includes pro-bullying and pro-victim attitudes and the reaction of teachers, peers, and parents to the bullying behavior (35). The Retrospective bullying experience addresses the adult population. It measures the frequency, significance, and extent of bullyvictimization. Important aspects like bully-related psychological trauma, suicidal ideation if bullied, and bullying in college and the workplace are also addressed (36).

Life events will be recorded using the modified HolmesRahe stress scale (41). This inventory includes 50 stressful events adapted to the cultural and social context of the Iranian population that are evaluated by Yes/No questions. The Internalized Stigma of Mental Illness scale (ISMI), a self-report questionnaire measuring self-stigma among persons with psychiatric disorders (42), will also be used. The Multidimensional Scale of Perceived Social Support (43) will evaluate the extent of support from family, friends, or significant people in patients' lives.

Religiosity will be measured using a scale based on Stark and Glock's dimensions of religiosity. This scale is adapted to 
TABLE 1 | Different domains of data collection at baseline and at 1, 3 and 5 years follow-up.

\begin{tabular}{|c|c|}
\hline Outcome parameter & Instrument \\
\hline Diagnosis (stability) & Structured psychiatric interview \\
\hline Symptom severity & $\begin{array}{l}\text { Positive and Negative Syndrome Scale, } \\
\text { Dimensions of Psychosis symptom severity } \\
\text { dimension tool of DSM-5 }\end{array}$ \\
\hline Depressive symptoms & Calgary Depression Scale in Schizophrenia \\
\hline Medication side effect & $\begin{array}{l}\text { St. Hans Rating Scale, Subjective Response to } \\
\text { Antipsychotics-34 }\end{array}$ \\
\hline Metabolic profile & Laboratory testing, anthropometrics \\
\hline \multicolumn{2}{|l|}{ Cognitive outcome } \\
\hline Working memory & $\begin{array}{l}\text { Forward digit span task, Backward digit span } \\
\text { task, Letter-number sequencing task, stroop task }\end{array}$ \\
\hline Attention & Trail making task \\
\hline $\begin{array}{l}\text { Visual and auditory memory } \\
\text { (immediate-delayed), recognition, } \\
\text { retention, and learning }\end{array}$ & $\begin{array}{l}\text { Auditory verbal learning test, Rey Osterrieth } \\
\text { complex figure }\end{array}$ \\
\hline Executive functioning & $\begin{array}{l}\text { Symbol digit modality task, letter digit modality } \\
\text { task, Verbal fluency task }\end{array}$ \\
\hline Speed of processing & Stroop task \\
\hline Intelligence quotation & Wechsler intelligence scale IV \\
\hline Social cognition & Benton Facial Recognition Test \\
\hline Theory of mind & Sali Ann \\
\hline \multicolumn{2}{|l|}{ Functional outcome } \\
\hline Disability & WHO Disability Assessment Schedule \\
\hline Functional remission & Functional remission tool \\
\hline Quality of life & Manchester Short Assessment of Quality of Life \\
\hline $\begin{array}{l}\text { Social, occupational, and } \\
\text { psychological functioning }\end{array}$ & Global Assessment of Functioning \\
\hline
\end{tabular}

the Islamic religion measuring dimensions of religious beliefs, practice, experience, and consequences with 26 questions (44).

Assessment of functioning will include (i) WHO Disability Assessment Schedule (WHODAS 2.0) (45), a standard tool applicable to clinical and general populations that covers six dimensions of cognition, mobility, self-care, getting along, life activities, and participation; (41) (ii) Global Assessment of Functioning (GAF) Scale form DSM (1) and (iii) the three-item Functional Recovery Tool including daily living and self-care, work, study, and housekeeping, and social contacts $(46,47)$. The Manchester Short Assessment of Quality of Life (MANSA) (48) will be used to assess satisfaction with life as a whole and with several domains like leisure, relationships, and mental health (42).

\section{Cognitive Assessments}

A comprehensive neurocognitive battery has been designed following the recommendations of the Measurement and Treatment Research to Improve Cognition in Schizophrenia (MATRICS) initiative (49), including eight cognitive domains (see Table 1).

Working memory will be tested with the forward and backward digit span and letter-number sequencing tasks (50). Divided attention, which is closely tied to executive functioning (51), will be assessed using the comprehensive (part B) test of the Trail making task. Verbal memory will be tested using The Rey Auditory Verbal Learning Test, measuring memory span, new learning, retention, recognition, and delayed recall (52). Visual learning will be measured using the Rey-Osterrieth Complex Figure. Executive functioning will be evaluated using the symbol digit modality task, letter digit modality task, backward digit span, and letter-number sequencing task. Speed of information processing will be measured with the average score of both the basic (part A) tests of Stroop (53) and Trail making (54), measured as time to complete in seconds, as well as category fluency by naming items from three different categories. The Stroop test will also give an estimation of inhibition and flexibility. Intelligence will be tested using the Wechsler Intelligence Scale-IV short version (55), validated for the Iranian population. Regarding social cognition, emotional recognition will be evaluated using the Reading the mind in the eyes test (56). The Benton Facial Recognition Test will be used to evaluate performance in face discrimination. Theory of mind will be tested using a Sally-Ann story in the Farsi language.

\section{Blood Sampling}

A trained nurse will collect $10 \mathrm{~mL}$ venous blood samples in EDTA (ethylenediaminetetraacetic acid) tubes utilizing a standard venipuncture at the local hospital laboratory. An individual specific barcode for each patient will be automatically assigned to each tube. This scannable code is unique for every study subject and does not contain elements of their demographics. The code will identify study subjects throughout documentation and evaluation and will be traceable only by the principal investigators. The biobank, located at the study coordinating center, will be monitored by the principal investigator.

Separate samples of whole blood, red blood cells, buffy coat, plasma, and serum will be coded and stored in freezers at $-80^{\circ} \mathrm{C}$ available at the sampling site until further analysis.

\section{Follow-Up}

All of the described assessments will be repeated during followup visits at 1, 3, and 5 years. Relevant outcomes will be measured on three different domains of (i) clinical outcome (i.e., symptoms, treatment efficacy, tolerability and cost, diagnostic stability), (ii) cognition, and (iii) social functioning and recovery (Table 1).

Patients will be invited and interviewed again to assess diagnostic stability and all symptoms will be recorded. Other assessments will include all of the assessments of the inclusion phase, any change in living situation and functioning of the patient, performance on neuro- and social cognition tests, function and disability, perceived stigma, components of metabolic disturbances, and all-cause mortality. Number of relapses, referral to the mental health care system, and rehospitalization will be recorded.

Based on the experience of a large cohort study in our region (57), several contact information (at least three) were gathered in the time of inclusion. Along with a routine synchronization with entry of the referral mental hospital of the province, this process was found to be the best solution for the best possible rate of retention. 


\section{Data Analysis}

Primary outcomes will be measured in two categories, symptom remission, including cognitive performance, and functional remission. The analysis will be performed to relate risk/resilience factors to the outcome measures and evaluate the course of the outcome. We considered five types of outcomes: (1) dimension of psychosis (58), (2) cognitive functioning (58), (3) symptom remission (59), (4) diagnostic stability (60), and (5) functional remission (61). Based on rates reported by previous studies, a minimum number was calculated to be 374 patients. Allowing for dropouts during follow-up, we estimated the total sample size to be 500 (please see sample power analysis).

Ratings will be performed by one trained psychologist. In case of multiple raters (lie collaboration of other refereal centers in other provinces), inter-rater concordance will be examined for observer-rated tools by suitable Kappa statistics. Raw data will be checked for invalid or nonsense entries. Some raw data will be coded into composite measures and data for investigators. For example, each component of the cognitive battery might have several tasks and repetitions. These multiple results will be combined to give a summary score based on the structure of the battery. A similar process is also applicable to other measures. Double-checking of data entry is performed by a trained psychologist who is not involved in patient inclusion. Digital data are checked weekly and compared to paper record, as well as any discrepancy between test results. Disagreements are then flagged and resolved.

Descriptive statistics using tabulations and graphical methods will be performed to achieve the objective I, after doublechecking data entry, data cleaning, and checking for consistency between different types of measurements. Neuropsychological test scores will be converted to domain $\mathrm{z}$ scores for correlational analysis to determine predictors of decline or reserve. For other questionnaires, there are predefined values for interpretation. After identifying the distribution of the data, kappa coefficients, correlations, multilevel regression, multidimensional analysis, cluster analysis, and longitudinal trajectory analysis will be used.

The magnitude of the difference between risk and resilience factors will be evaluated by comparing standardized mean differences to achieve objective II. The relationship between risk and resilience factors will be assessed by logistic regression (for binary outcomes) and multivariable linear regressions (for continuous normally distributed outcomes). Appropriate longitudinal data methods will be used for the assessment of disease progression from baseline first episode status to disease at follow-up in years 1, 3, and 5. Multivariable models will determine risk factors that predict cognitive, personal, and clinical outcomes, when appropriate. Multilevel analysis to compare disease progression will also be considered.

We will perform a cluster analysis to achieve objective III. The hierarchical cluster method will be applied to identify the number of clusters for each outcome and seed points for a k-means cluster analysis. As an example, neurocognitive subtypes will be compared on baseline clinical and socio-demographic variables by conducting Chi-square analyses or Kruskal Wallis tests on gender, age, social support, educational level, substance use, schizophrenia spectrum disorder diagnosis, symptom severity, dosage of antipsychotic medication, and adverse life events.

We will define predictive models for objective IV to assess the predictive value of (using the previous example) cognition on symptomatic and functional outcomes at followup. Separate clinically and statistically meaningful trajectories will be identified using censored normal group-based trajectory modeling (62) and evaluated for model classification accuracy. Predicted trajectories will be evaluated using a random-effect ordinal regression analysis.

Power calculation: In a recent study [(63); Submitted] in the Dutch population, we observed three symptoms trajectories of positive symptoms with a frequency of $67.8,23.3$, and $8.9 \%$, with a range of significantly associated predictors of effect size (odds ratio), range 1.2-1.8, obtained through multinominal regression models. Translating these observations to the ARAS study consisting of 500 subjects, we expected to observe three groups of patients' clinical trajectories, respectively consisting of $340\left(0.68^{*} 500\right), 115\left(0,23^{*} 500\right)$, and 45 subjects. Give the study includes three-time points of T0, T3, and T5, we consider a simple repeated data scenario to estimate an expected power of estimate: assuming b/a ratio of $3, \mathrm{a}=0.05$, beta 0.20 , one main predictor of two levels partialized on the three trajectories (outcome levels: disease course) and thus a $3 * 2$ design consisting of 6 groups, and 3 covariables, using F-test for ANCOVA for repeated measures analysis; we will have a power of 0.63 to detect small effect $(f)$ size of 0.15 at a $p$-value of $0.05,0.86$ to detect a medium $f$ of 0.25 ; and full power to detect a large $f$ of 0.40 at a $p$-value of $10^{-6}$. Thus, ARAS study will offer sufficient power to detect factors with medium and large effects on various multilevels analyses. We used $\mathrm{G}^{*}$ power ver 3 for power calculation.

\section{DISCUSSION}

The ARAS cohort study will have additive value by characterizing SSD and other psychotic disorders in a population living in the Middle East. Given the extensive differences between various aspects of life in Middle Eastern and Western countries, it is highly expected that the ARAS will offer a novel understanding of disease mechanisms and outcomes in SSD. However, the most important novel aspect is the study population. Several differences are hypothetically present when available studies are compared to our population. Iranian patients may not only have a slightly different genetic susceptibility, especially concerning population-specific rare genetic variants compared to other countries, but they live in quite different environmental conditions for social support, public stressors, religion, culture, eating habits, inequality, lifestyle, and the other risk/resilience factors that may influence the course of the disorder. This diversity provides valuable and rich untouched research material. A first example is illicit drugs (64), as there is an obvious difference between Iran, with opioids as the most common drug, and European countries, where cannabis use is more prevalent. Another example would be different lifestyles and diet-intakes that putatively influence the emergence of metabolic disturbances in patients with psychotic disorders (65). The ARAS 
study is also the first study on the quality of care for patients with SSD in Iran. Gathering information on a wide variety of treatments, the project per se will improve the quality of care for participating patients and can stand as the first step in this pathway.

The project might face several limitations that come with longitudinal natural cohort studies. Data collection may be hampered by the duration of the diagnostic battery and arrangements. Training of the interviewers and use of standardized procedures for data collection is expected to contribute to a low proportion of missing data, and no imputation is being planned. Patients may be lost to followup because of e.g., distance, stigma, and lack of insight. Incomplete data from illiterate patients is another challenge; however, illiteracy will not interfere with many aspects of evaluations, such as the diagnostic interview and several parts of the cognitive battery as well as the expected outcome. On the other hand, improvement in the care provided during the project might increase the cooperation of patients and their families.

In conclusion, the ARAS cohort will yield significant academic- (research and education) and care-related achievements. This study will provide novel data about risk and resilience factors for SSD. The National health care system will benefit from reliable data on care for patients with SSD. ARAS data and experience will have value both in being a useful model for other parts of this region and in the expansion of the currently available knowledge. Eventually, ARAS will help to

\section{REFERENCES}

1. American Psychiatric Association. Diagnostic and Statistical Manual of Mental Disorders (DSM-5 $\left.{ }^{\circledR}\right)$. Washington, DC: American Psychiatric Pub (2013). doi: 10.1176/appi.books.9780890425596

2. Millier A, Schmidt U, Angermeyer MC, Chauhan D, Murthy V, Toumi M, et al. Humanistic burden in schizophrenia: a literature review. J Psychiatr Res. (2014) 54:85-93. doi: 10.1016/j.jpsychires.2014. 03.021

3. Wu EQ, Birnbaum HG, Shi L, Ball DE, Kessler RC, Moulis M, et al. The economic burden of schizophrenia in the United States in 2002. J Clin Psychiatry. (2005) 66:1122-9. doi: 10.4088/JCP.v66 n0906

4. Schofield P, Das-Munshi J, Becares L, Agerbo E. Neighbourhood ethnic density and incidence of psychosis-First and second generation migrants compared. European Psychiatry. (2017) 41:S249. doi: 10.1016/j.eurpsy.2017.02.034

5. Muli A, Sideli L, Capuccio V, Fearon P, Ferraro L, Kirkbride JB, et al. Low incidence of psychosis in Italy: confirmation from the first epidemiological study in Sicily. Soc Psychiatry Psychiatr Epidemiol. (2017) 52:155-62. doi: 10.1007/s00127-016-1322-4

6. McGrath J, Saha S, Welham J, El Saadi O, MacCauley C, Chant D. A systematic review of the incidence of schizophrenia: the distribution of rates and the influence of sex, urbanicity, migrant status and methodology. BMC Med. (2004) 2:13. doi: 10.1186/1741-7015-2-13

7. Kirkbride JB, Fearon P, Morgan C, Dazzan P, Morgan K, Tarrant J, et al. Heterogeneity in incidence rates of schizophrenia and other psychotic syndromes: findings from the 3-center AeSOP study. Arch Gen Psychiatry. (2006) 63:250-8. doi: 10.1001/archpsyc.63.3.250

8. Perälä J, Suvisaari J, Saarni SI, Kuoppasalmi K, Isometsä E, Pirkola S, et al. Lifetime prevalence of psychotic and bipolar i optimize healthcare for people with SSD and improve the quality of life of this vulnerable group of people.

\section{DATA AVAILABILITY STATEMENT}

The original contributions presented in the study are included in the article/supplementary material, further inquiries can be directed to the corresponding author/s.

\section{AUTHOR CONTRIBUTIONS}

SF drafted the manuscript and it was modified by by all authors. $\mathrm{RB}, \mathrm{BA}$, and SF conceived the study and were major contributors to writing the manuscript. WV, MG, AM, MS, and AS-K contributed to the design and study protocol. All authors read and approved the final manuscript.

\section{FUNDING}

This study was funded by the National Institute for Medical Research Development (NIMAD), Iran. The funding body has no role in the design of the study and writing the manuscript.

\section{ACKNOWLEDGMENTS}

We are grateful to the colleagues and the Research development unit of Razi hospital at Tabriz university of medical sciences for supporting this study.

disorders in a general population. Arch Gen Psychiatry. (2007) 64:19-28. doi: 10.1001/archpsyc.64.1.19

9. McGrath J, Saha S, Chant D, Welham J. Schizophrenia: a concise overview of incidence, prevalence, and mortality. Epidemiol Rev. (2008) 30:67-76. doi: 10.1093/epirev/mxn001

10. Sharifi V, Kermani-Ranjbar T, Amini H, Alaghband-rad J, Salesian N, Seddigh A. Duration of untreated psychosis and pathways to care in patients with first-episode psychosis in Iran. Early Intervent Psychiatry. (2009) 3:1316. doi: 10.1111/j.1751-7893.2009.00119.x

11. Susser E, Martinez-Alis G. Putting psychosis into sociocultural context: an international study in 17 locations. JAMA Psychiatry. (2018) 75:910. doi: 10.1001/jamapsychiatry.2017.3541

12. Islam MA, Khan MH, Quee PJ, Snieder H, van den Heuvel ER, Bruggeman R, et al. Familial liability to psychosis is a risk factor for multimorbidity in people with psychotic disorders and their unaffected siblings. Europ Psychiatry. (2017) 45:81-9. doi: 10.1016/j.eurpsy.2017.05.001

13. Bernardo M, Bioque M, Cabrera B, Lobo A, González-Pinto A, Pina L, et al. Modelling gene-environment interaction in first episodes of psychosis. Schizophr Res. (2017) 189:181-9. doi: 10.1016/j.schres.2017.01.058

14. Zwicker A, Denovan-Wright EM, Uher R. Gene-environment interplay in the etiology of psychosis. Psychol Med. (2018) 48:1925-36. doi: 10.1017/S003329171700383X

15. Pekkanen J, Pearce N. Environmental epidemiology: challenges and opportunities. Environ Health Perspect. (2001) 109:1-5. doi: 10.2307/3434913

16. Holt RIG, Peveler RC, Byrne CD. Schizophrenia, the metabolic syndrome and diabetes. Diabetic Med. (2004) 21:51523. doi: 10.1111/j.1464-5491.2004.01199.x

17. Rady MY, Johnson DJ, Patel BM, Larson JS, Helmers RA. Influence of individual characteristics on outcome of glycemic control in intensive care unit patients with or without diabetes mellitus. Mayo Clin Proc. (2005) 80:1558-67. doi: 10.4065/80.12.1558 
18. Fadai F, Mousavi B, Ashtari Z, Farhang S, Hashempour S, Shahhamzei $\mathrm{N}$, et al. Saffron aqueous extract prevents metabolic syndrome in patients with schizophrenia on olanzapine treatment: a randomized triple blind placebo controlled study. Pharmacopsychiatry. (2014) 47:15661. doi: 10.1055/s-0034-1382001

19. Lieberman JA, Tollefson G, Tohen M, Green AI, Gur RE, Kahn R, et al. Comparative efficacy and safety of atypical and conventional antipsychotic drugs in first-episode psychosis: a randomized, double-blind trial of olanzapine versus haloperidol. Am J Psychiatry. (2003) 160:1396404. doi: 10.1176/appi.ajp.160.8.1396

20. Menezes NM, Arenovich T, Zipursky RB. A systematic review of longitudinal outcome studies of first-episode psychosis. Psychol Med. (2006) 36:134962. doi: 10.1017/S0033291706007951

21. Hwang R, Zai C, Tiwari A, Muller DJ, Arranz MJ, Morris AG, et al. Effect of dopamine D3 receptor gene polymorphisms and clozapine treatment response: exploratory analysis of nine polymorphisms and meta-analysis of the Ser9Gly variant. Pharmacogenom J. (2010) 10:200. doi: 10.1038/tpj.2009.65

22. An SK, Kang JI, Park JY, Kim KR, Lee SY, Lee E. Attribution bias in ultrahigh risk for psychosis and first-episode schizophrenia. Schizophr Res. (2010) 118:54-61. doi: 10.1016/j.schres.2010.01.025

23. Yamazawa R, Nemoto T, Kobayashi H, Chino B, Kashima H, Mizuno M. Association between duration of untreated psychosis, premorbid functioning, and cognitive performance and the outcome of first-episode schizophrenia in Japanese patients: prospective study. Austr N Zealand J Psychiatry. (2008) 42:159-65. doi: 10.1080/00048670701787537

24. World Health Organization, World Organization of National Colleges, Academies, Academic Associations of General Practitioners/Family Physicians. Integrating Mental Health Into Primary Care: A Global Perspective. Tehran: World Health Organization (2008).

25. Rezaei O, Khodaie-Ardakani M, Mandegar MH, Dogmehchi E, Goodarzynejad H. Prevalence of metabolic syndrome among an Iranian cohort of inpatients with schizophrenia. Int J Psychiatry Med. (2009) 39:451-62. doi: 10.2190/PM.39.4.i

26. Sadeghieh Ahari S, Nikpou H, Molavi P, Abdi R, Amani F, Shirinzadeh B. An investigation of duration of untreated psychosis and the affecting factors. $J$ Psychiatr Ment Health Nurs. (2014) 21:87-92. doi: 10.1111/jpm.12067

27. Goreishizadeh MA, Mohagheghi A, Farhang S, Alizadeh L. Psychosocial disabilities in patients with schizophrenia. Iran J Public Health. (2012) 41:116.

28. Shamsaei F, Cheraghi F, Bashirian S. Burden on family caregivers caring for patients with schizophrenia. Iran J Psychiatry. (2015) 10:239-45.

29. Shooshtari MH, Davari-Ashtiani R, Shahrivar Z, Shabani A, Semnani Y, Kaviani H, et al. Structured clinical interview for DSM-IV (SCID Persian translation and cultural adaptation). Iran J Psychiatry. (2007) 2:46-8.

30. Ghanizadeh A, Mohammadi MR, Yazdanshenas A. Psychometric properties of the Farsi translation of the kiddie schedule for affective disorders and schizophrenia-present and lifetime version. BMC Psychiatry. (2006) 6:10. doi: 10.1186/1471-244X-6-10

31. Yousefi-Nooraie R, Mohammadi MR, Salesian N, Amin-Esmaeeli M, Mansouri N, Mesgarpour B, et al. Prevalence of psychiatric disorders in Iran: a systematic review. Iran J Psychiatry. (2007) 2:137-50.

32. Tandon R, Gaebel W, Barch DM, Bustillo J, Gur RE, Heckers S, et al. Definition and description of schizophrenia in the DSM-5. Schizophr Res. (2013) 150:3-10. doi: 10.1016/j.schres.2013.05.028

33. Kay SR, Fiszbein A, Opfer LA. The positive and negative syndrome scale (PANSS) for schizophrenia. Schizophr Bull. (1987) 13:261. doi: 10.1093/schbul/13.2.261

34. Kontaxakis VP, Havaki-Kontaxaki BJ, Stamouli SS, Margariti MM, Collias CT, Christodoulou GN. Comparison of four scales measuring depression in schizophrenic inpatients. European Psychiatry. (2000) 15:274-7. doi: 10.1016/S0924-9338(00)00232-7

35. Addington D, Addington J, Maticka-Tyndale E. Assessing depression in schizophrenia: the calgary depression scale. Br J Psychiatry. (1993) 22:3944. doi: 10.1192/S0007125000292581

36. A Wolters H, Knegtering R, Wiersma D, J van dB. Evaluation of the subjects' response to antipsychotics questionnaire. Int Clin Psychopharmacol. (2006) 21:63-9. doi: 10.1037/t70822-000

37. Gerlach J, Korsgaard S, Clemmesen P, Lauersen AL, Magelund G, Noring $U$, et al. The St. Hans Rating Scale for extrapyramidal syndromes: reliability and validity. Acta Psychiatr Scand. (1993) 87:244-52. doi: 10.1111/j.1600-0447.1993.tb03366.x

38. Akbari Balootbangan A, Talepasand S. Validation of the Illinois bullying scale in primary school students of Semnan, Iran. J Fundamentals Mental Health. (2015) 17:11-2.

39. Rezapour M, Soori H, Khodakarim S. Testing psychometric properties of the perpetration of bullying and victimization scales with olweus bullying questionnaire in middle schools. J Safety Promot Injury Prevent. (2014) 1:21221.

40. Schäfer M, Korn S, Smith PK, Hunter SC, Mora-Merchán JA, Singer MM, et al. Lonely in the crowd: recollections of bullying. Br J Dev Psychol. (2004) 22:379-94. doi: 10.1348/0261510041552756

41. Malek A, Poorafkary N, Dadashzadeh H, Safaeeyan A. Ranking of stressful life events in general population of Tabriz, Iran. Res J Biol Sci. (2008) 3:993-8.

42. Ritsher JB, Otilingam PG, Grajales M. Internalized stigma of mental illness: psychometric properties of a new measure. Psychiatry Res. (2003) 121:3149. doi: 10.1016/j.psychres.2003.08.008

43. Zimet GD, Dahlem NW, Zimet SG, Farley GK. The multidimensional scale of perceived social support. J Pers Assess. (1988) 52:3041. doi: 10.1207/s15327752jpa5201_2

44. Khodayarifard M, Ghorbani B, Paknejad M, Rahiminezhad A. Religiosity measuring model and preparation of religiosity scale to Iranian population. J Applied Psychol Res. (2001) 11:1-24.

45. Ustun TB. Measuring Health and Disability: Manual for WHO Disability Assessment Schedule WHODAS 2.0. Geneva: World Health Organization (2010).

46. Wiersma D, Visser E, Bähler M, Bruggeman R, Delespaul PA, Keet IP, et al. Functional remission of people with serious mental illness (SMI): psychometric properties of a new ROM-instrument. Tijdschrift voor Psychiatrie. (2015) 57:395-404.

47. Swildens WE, Visser E, Bähler M, Bruggeman R, Delespaul P, van der Gaag $\mathrm{M}$, et al. Functional recovery of individuals with serious mental illnesses: Development and testing of a new short instrument for routine outcome monitoring. Psychiatr Rehabil J. (2018) 41:341. doi: 10.1037/prj0000320

48. Bjorkman T, Svensson B. Quality of life in people with severe mental illness. reliability and validity of the Manchester Short Assessment of Quality of Life (MANSA). Nordic J Psychiatry. (2005) 59:302-6. doi: 10.1080/08039480500213733

49. Arranz MJ, De Leon J. Pharmacogenetics and pharmacogenomics of schizophrenia: a review of last decade of research. Mol Psychiatry. (2007) 12:707-47. doi: 10.1038/sj.mp.4002009

50. Crowe SF. Does the letter number sequencing task measure anything more than digit span? Assessment. (2000) 7:1137. doi: $10.1177 / 107319110000700202$

51. Salthouse TA. What cognitive abilities are involved in trail-making performance? Intelligence. (2011) 39:222-32. doi: 10.1016/j.intell.2011.03.001

52. Rezvanfard M, Ekhtiari H, Noroozian M. The rey auditory verbal learning test: alternate forms equivalency and reliability for the Iranian adult population (Persian version). Arch Iran Med. (2011) 14:104.

53. Stroop JR. Studies of interference in serial verbal reactions. J Exp Psychol. (1935) 18:643. doi: 10.1037/h0054651

54. Strauss E, Sherman EMS, Spreen O. A Compendium of Neuropsychological Tests: Administration, Norms, and Commentary. New York, NY: Oxford University Press (2006)

55. Reza JA, Abas P. Reliability and validity of wechsler intelligence scale for childrenthird edition (WISC-III) in Iran. J Med Educ. (2003) 2:75-80.

56. Khorashad BS, Baron-Cohen S, Roshan GM, Kazemian M, Khazai L, Aghili Z, et al. The "Reading the Mind in the Eyes" test: investigation of psychometric properties and test-retest reliability of the persian version. J Autism Dev Disord. (2015) 45:2651-66. doi: 10.1007/s10803-015-2427-4

57. Farhang S, Faramarzi E, Amini Sani N, Poustchi H, Ostadrahimi A, Alizadeh BZ, et al. Cohort profile: the AZAR cohort, a health-oriented research model in areas of major environmental change in Central Asia. Int J Epidemiol. (2018) 48:382-382h. doi: 10.1093/ije/dyy215

58. Albert N, Melau M, Jensen H, Hastrup LH, Hjorthøj C, Nordentoft M. The effect of duration of untreated psychosis and treatment delay on the outcomes of prolonged early intervention in psychotic disorders. NPJ Schizophr. (2017) 3:34. doi: 10.1038/s41537-017-0034-4 
59. Lally J, Ajnakina O, Stubbs B, Cullinane M, Murphy KC, Gaughran F, et al. Remission and recovery from first-episode psychosis in adults: systematic review and meta-analysis of long-term outcome studies. Br J Psychiatry. (2017) 211:350-8. doi: 10.1192/bjp.bp.117.201475

60. Pope MA, Joober R, Malla AK. Diagnostic stability of first-episode psychotic disorders and persistence of comorbid psychiatric disorders over 1 year. Canad J Psychiatry. (2013) 58:588-94. doi: 10.1177/070674371305801008

61. Verma S, Subramaniam M, Abdin E, Poon LY, Chong SA. Symptomatic and functional remission in patients with first-episode psychosis. Acta Psychiatr Scand. (2012) 126:282-9. doi: 10.1111/j.1600-0447.2012.01883.x

62. Jones BL, Nagin DS, Roeder K. A SAS procedure based on mixture models for estimating developmental trajectories. Sociol Methods Res. (2001) 29:37493. doi: 10.1177/0049124101029003005

63. Habtewold TD, Liemburg EJ, Islam MA, de Zwarte SM, Boezen HM, Luykx JJ, et al. Association of schizophrenia polygenic risk score with datadriven cognitive subtypes: a six-year longitudinal study in patients, siblings and controls. Schizophr Res. (2020) 223:135-47. doi: 10.1016/j.schres.2020. 05.020
64. Semple DM, McIntosh AM, Lawrie SM. Cannabis as a risk factor for psychosis: systematic review. J Psychopharmacol. (2005) 19:18794. doi: $10.1177 / 0269881105049040$

65. Papanastasiou E. The prevalence and mechanisms of metabolic syndrome in schizophrenia: a review. Therap Adv Psychopharmacol. (2013) 3:3351. doi: $10.1177 / 2045125312464385$

Conflict of Interest: The authors declare that the research was conducted in the absence of any commercial or financial relationships that could be construed as a potential conflict of interest.

Copyright (C) 2021 Farhang, Ghaemmaghami, Shafiee-Kandjani, Noorazar, Veling, Malek, Somi, Bruggeman and Alizadeh. This is an open-access article distributed under the terms of the Creative Commons Attribution License (CC BY). The use, distribution or reproduction in other forums is permitted, provided the original author(s) and the copyright owner(s) are credited and that the original publication in this journal is cited, in accordance with accepted academic practice. No use, distribution or reproduction is permitted which does not comply with these terms. 